\title{
EEG profiles during general anesthesia in children: $A$ comparative study between sevoflurane and propofol
}

\section{Agnes Rigouzzo ${ }^{1}$ (D) | Linda Khoy-Ear ${ }^{1}$ | Dominique Laude ${ }^{2}$ (D) $\mid$ Nicolas Louvet $^{1}$ | Marie-Laure Moutard $^{3}$ | Nada Sabourdin ${ }^{1}$ | Isabelle Constant ${ }^{1}$}

${ }^{1}$ Anesthesiology Department, Hôpital Armand Trousseau, Paris, France

${ }^{2}$ Inserm U1138, Université Pierre \& Marie Curie Paris 6, Paris, France

${ }^{3}$ Service de Neuropédiatrie, Hôpital Armand Trousseau, Paris, France

\section{Correspondence}

Agnes Rigouzzo, Anesthesiology

Department, Armand Trousseau Children

Hospital, Paris, France.

Email: agnes.rigouzzo@aphp.fr

Section Editor: Dean Kurth

\begin{abstract}
Background: In this prospective study, we describe the electroencephalographic (EEG) profiles in children anesthetized with sevoflurane or propofol.

Methods: Seventy-three subjects (11 years, range 5-18) were included and randomly assigned to two groups according to the anesthetic agent. Anesthesia was performed by target-controlled infusion of propofol (group P) or by sevoflurane inhalation (group S). Steady-state periods were performed at a fixed randomized concentration between 2, 3, 4, 5, and $6 \mu \mathrm{g} \cdot \mathrm{ml}^{-1}$ of propofol in group $\mathrm{P}$ and between $1,2,3,4$, and $5 \%$ of sevoflurane in group S. Remifentanil was continuously administered throughout the study. Clinical data, Bispectral Index (BIS), and raw EEG were continuously recorded. The relationship between BIS and anesthetic concentrations was studied using nonlinear regression. For all steady-state periods, EEG traces were reviewed to assess the presence of epileptoid signs, and spectral analysis of raw EEG was performed.

Results: Under propofol, BIS decreased monotonically and EEG slowed down as concentrations increased from 2 to $6 \mu \mathrm{g} \cdot \mathrm{ml}^{-1}$. Under sevoflurane, BIS decreased from $0 \%$ to $4 \%$ and paradoxically rose from $4 \%$ to $5 \%$ of expired concentration: this increase in BIS was associated with the occurrence of fast oscillations and epileptoid signs on the EEG trace. Propofol was associated with more delta waves and burst suppression periods compared to sevoflurane.

Conclusion: Under deep anesthesia, the BIS and electroencephalographic profiles differ between propofol and sevoflurane. For high concentrations of sevoflurane, an elevated BIS value may be interpreted as a sign of epileptoid patterns or EEG fast oscillations rather than an insufficient depth of hypnosis.

KEYWORDS

EEG, general anesthesia, pharmacodynamics, sevoflurane, TIVA
\end{abstract}

\section{1 | INTRODUCTION}

Childhood is characterized by numerous physiological changes linked to growth. Some of these changes might affect the anesthetic management. For example, the brain maturation progresses with age; and the pharmacologic properties of anesthetic agents also change with age. As a consequence, the interindividual variability of anesthetics requirement is majored in the pediatric population. Because of this variability, it seems particularly important to have a pharmacodynamic feedback of the cerebral effects of our drugs. Such feedback might allow a more accurate and individualized drug delivery. 
The cerebral effects of anesthetics may be monitored by analyzing the cortical EEG signal. Age-dependent EEG profiles have been widely described in non-anesthetized children. In contrast, EEG data in anesthetized children are scarce. Among the few available data, epileptiform EEG has been described by some authors for high concentrations of sevoflurane. ${ }^{1,2}$ In addition, recent publications using spectral and coherence methods have evidenced age-related differences in EEG profiles during surgical anesthesia. ${ }^{3,4}$ However, the potential drug-specific, dose-related modifications remain poorly investigated.

Despite the few available EEG data, there are many pediatric publications focusing on EEG-based monitors. In these studies, the Bispectral Index (BIS), which provides a single number calculated from an algorithm involving cortical EEG parameters, is one of the most studied indices.

Sevoflurane and propofol are the most commonly used hypnotic drugs in children. The dose-dependent BIS profile in children receiving these agents has been described by several authors:

- The BIS is inversely correlated with the dose of propofol. ${ }^{5}$ No dose-related raw EEG pediatric analyses are available to explain this relationship, except for some data describing a decrease in spectral edge frequency for increasing doses of propofol. ${ }^{6}$

- Under sevoflurane, several studies describe a steady decrease in BIS for increasing sevoflurane concentrations between $1 \%$ and $3 \%{ }^{7,8}$ As for propofol, no dose-related raw EEG pediatric analyses are available to explain this relationship, except for some data describing a decrease in spectral edge frequency for increasing doses of sevoflurane. ${ }^{9,10}$ For higher concentrations, some authors report cases of increase in BIS value. ${ }^{8,11}$ There is no clear explanation to this phenomenon, often qualified as "paradoxical."

This study was designed to describe more precisely the EEG characteristics in children anesthetized with different doses of propofol or sevoflurane, and how these EEG profiles are reflected by the BIS. Our first objective was to describe the dose-response relationship between BIS and propofol or sevoflurane concentrations. Our second objective was to study the raw EEG using spectral analysis and epileptoid sign screening, in order to describe the EEG features corresponding to the BIS changes. Finally, we compared our BIS and EEG findings, between propofol and sevoflurane at an equipotent level of cortical inhibition, corresponding to multiples of EC50 $\mathrm{BIS}$ calculated for each agent.

\section{2 | PATIENTS AND METHODS}

\section{1 | Patients and study design}

\subsection{1 | Patients}

This prospective randomized study was approved by our local Ethics Committee (CPP Saint-Antoine, Paris, France), and written and informed consent was obtained from children and their parents.

\section{What is already known}

- Sevoflurane and propofol have different electroencephalographic effects.

\section{What this article adds}

- Under deep anesthesia, the BIS profiles differ between propofol and sevoflurane.

Seventy-three children ranging from 5 to 18 years of age, ASA 1 or 2 , scheduled for middle ear surgery, were prospectively included.

The randomization was a two-step process, based on computergenerated lists. First, patients were assigned to either the propofol or the sevoflurane group. Then, within each group (sevoflurane/ propofol), the dose of the agent was randomized, with five possibilities in each group: Sevoflurane 1/2/3/4/5\%; Propofol 2/3/4/5/ $6 \mu \mathrm{g} \cdot \mathrm{ml}^{-1}$ (flowchart in Figure 1).

Exclusion criteria were: obesity, cardiac, pulmonary or renal disease; neurological or neuromuscular disorders; and preoperative administration of medications which interfere with the central nervous system.

\subsection{Study design}

Before surgery, all patients were premedicated with oral hydroxyzine (1 mg.kg-1).

Additionally to the standard monitoring, the disposable adult BisSensor (Aspect Medical Systems) was connected to a BIS monitor Cardiocapll (Datex-GE). The smoothing window was set at $15 \mathrm{~s}$.

\subsubsection{Sevoflurane (S) group}

Anesthesia was induced with sevoflurane $6 \%$ in $100 \%$ oxygen. At loss of eyelash reflex, remifentanil was started at $0.25 \mu \mathrm{g} . \mathrm{kg}^{-1} \cdot \mathrm{min}^{-1}$ and kept constant throughout the study. A single dose of atracurium (0.5 mg. $\left.\mathrm{kg}^{-1}\right)$ was administered and intubation was performed using a cuffed tracheal tube.

Until the end of surgery, mechanical ventilation was maintained with an air-oxygen mixture (50-50). Breathing frequency was set at a rate ranging from 14 to 20 breaths. $\mathrm{min}^{-1}$, according to age, and $\mathrm{ET}_{\mathrm{CO} 2}$ was kept strictly between 30 and $35 \mathrm{mmHg}$.

For each patient, before surgery and according to the constraints of operating schedule, two 10-min steady-state periods were performed at a fixed expired fraction of $\mathrm{S}(\mathrm{FeS})$ randomized between 1 , $2,3,4$, and $5 \%$.

\subsection{2 | Propofol (P) group}

Propofol was administered via a target-controlled infusion (TCl) device with a propofol plasma target concentration ( $C t P$ ) of6 $\mu \mathrm{g} \cdot \mathrm{ml}^{-1}$. The $\mathrm{TCl}$ system consisted of a Cardinal-Alaris infusion pomp 

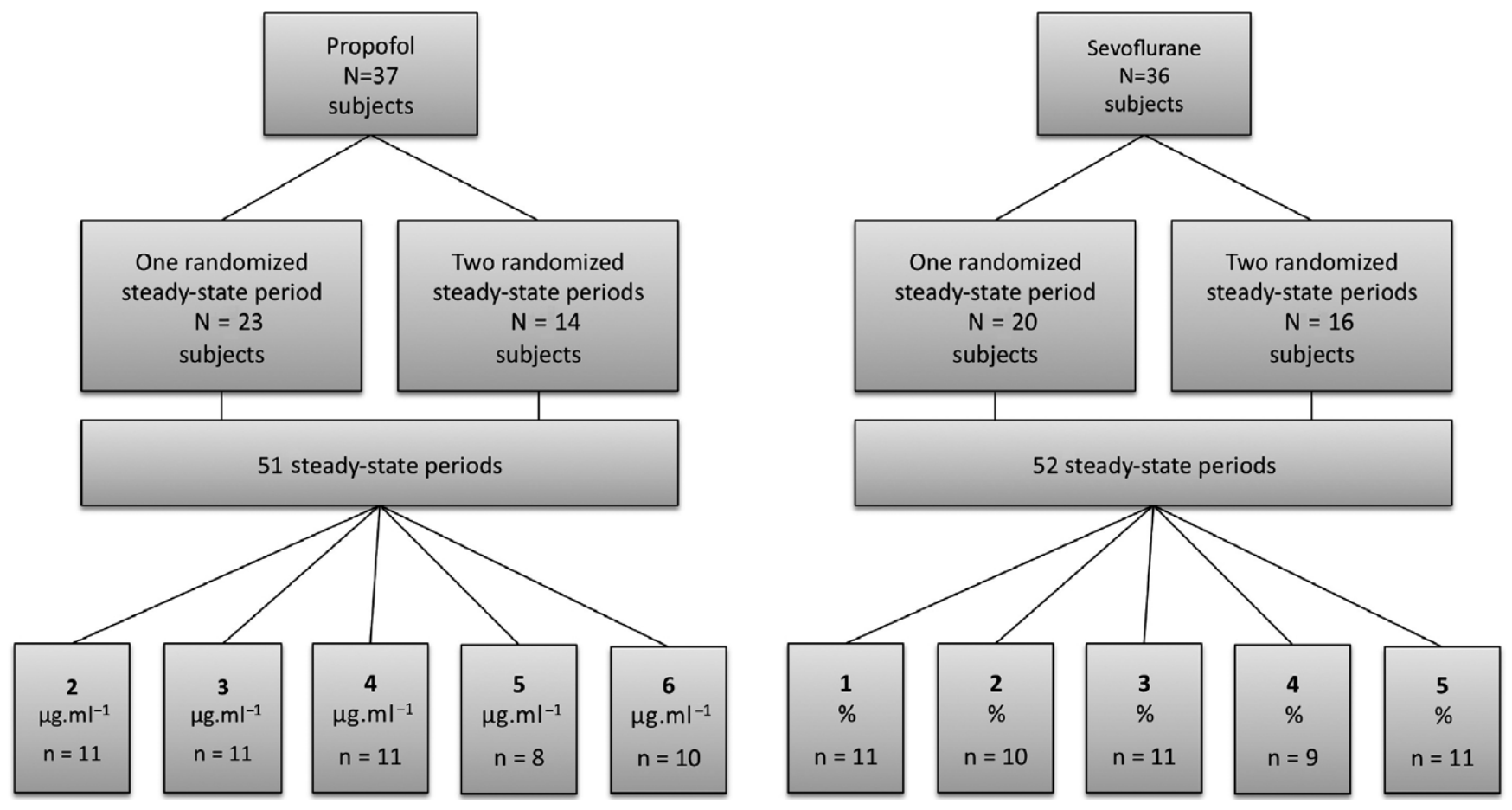

FIGURE 1 Flow chart of study design

driven by Rugloop software based on the Kataria's model for prepubertal children ${ }^{12}$ and on the Schnider's model for postpubertal teeangers. $^{13}$ At loss of eyelash reflex, remifentanil was started at $0.25 \mu \mathrm{g} \cdot \mathrm{kg}^{-1} \cdot \mathrm{min}^{-1}$ and kept constant throughout the study. A single dose of atracurium ( $\left.0.5 \mathrm{mg} . \mathrm{kg}^{-1}\right)$ was administered and intubation was performed using a cuffed tracheal tube. Mechanical ventilation was maintained with an air-oxygen mixture (50-50). Breathing frequency was fixed at a rate ranging from 14 to 20 breaths. $\mathrm{min}^{-1}$ according to age, and endtidal concentration of $\mathrm{CO} 2\left(\mathrm{ET}_{\mathrm{CO} 2}\right)$ was kept strictly between 30 and $35 \mathrm{mmHg}$.

For each patient, before surgery and according to the constraints of operating schedule, two 10-min steady-state periods were performed at a fixed $\mathrm{Ct} P$ randomized between 2, 3, 4, 5, and $6 \mu \mathrm{g} \cdot \mathrm{ml}^{-1}$.

All patients were interviewed on postoperative day 1, using a standardized questionnaire to detect intraoperative awareness.

\subsection{Data acquisition}

The following data were automatically and continuously recorded, with a sample rate of $4 \mathrm{~Hz}$, using the Rugloop II software loaded into a dedicated microcomputer:

- heart rate, noninvasive blood pressure, respiratory parameters, $\mathrm{SpO} 2$

- Expired gases: EtCO2, FiO2, FeS

- Infusion parameters: rate of propofol infusion, targeted and estimated concentrations of propofol
- BIS and suppression ratio

The EEG was continuously recorded independently from the BIS monitor. A single channel was recorded from $\mathrm{Ag} \pm \mathrm{AgCl}$ electrodes placed on the forehead and left mastoid, with the right mastoid as the common electrode. The electrode impedance was checked automatically and maintained below $5 \mathrm{~kW}$.

The EEG signal was acquired $(256 \mathrm{~Hz})$ on a microcomputer using the Brain-Quick program stem II (Micromed, Merignac, France). The bandpass filter was set at $0.5-30 \mathrm{~Hz}$.

\subsection{Data analysis}

\subsubsection{Analysis of BIS data}

The baseline value of BIS was calculated as the average value of BIS on the last minute before the beginning of induction of anesthesia. At each 10-min steady-state period corresponding to a randomized Fe $\mathrm{S}$ or $\mathrm{Ct} \mathrm{P}$, the average value of BIS was calculated on the last minute of the steady-state period. These parameters were expressed as mean $\pm \mathrm{SD}$.

The relationship between BIS and anesthetic concentrations was studied using nonlinear regression calculated on steady-state periods at fixed randomized FeS or CtP.

Then, the $\mathrm{EC} 5 \mathrm{~B}_{\mathrm{BIS}}$ corresponding to half-maximal effect (BIS 50) was calculated from the dose-response curve fitted from the data obtained for each agent. 
Each concentration of propofol and sevoflurane steady-state periods was then expressed as a multiple of the respective $\mathrm{EC}^{5} \mathrm{~B}_{\mathrm{BIS}}$, to define the level of BIS depression.

\subsection{2 | Analysis of EEG data}

\section{Epileptoid signs assessment}

EEG recordings during all steady-state periods were blindly analyzed and classified by two experts including one pediatric neurologist (MLM) and one pediatric anesthesiologist (IC) to assess whether seizure-like activity was present. Epileptiform activity was classified as suggested in previous publications ${ }^{14}$ : spike (Sp), spike and wave (Sw), polyspikes (PS), rhythmic polyspikes (RPS), rhythmic polyspikes and burst suppression (SBS), periodic epileptiform discharges (PED), and electrographic seizure (ES). The incidence of minor Epileptoid Signs (Sp, Sw, PS), and major Epileptoid Signs (RPS, SBS, PED, ES) was calculated for all steady-state periods.

\section{Spectral analysis}

The EEG was analyzed in the frequency domain using spectral analysis, performed with fast Fourier transformation (FFT) (Acqknowledge v3.25; Biopac Systems, Santa Barbara, CA, USA) on 8-s epoch. ${ }^{15}$ The total spectral power (TSP), defined as the area under the curve of the spectrum $\left(\mathrm{mV}^{2}\right)$ was calculated as the spectral power of the following bands:delta $(0-4 \mathrm{~Hz})$, theta $(4-8 \mathrm{~Hz})$, alpha $(8-13 \mathrm{~Hz})$, and beta (13-30 Hz). The results of this calculation were presented as the relative contribution of each band to the TSP, calculated as a percentage of TSP. These EEG-derived variables were calculated at baseline, and on the last minute of each steady-state period.

\section{5 | Statistical analysis}

Results were expressed as mean \pm SD. Statistical differences in population data between $\mathrm{S}$ and $\mathrm{P}$ groups were investigated using a Mann-Whitney test.

Relationship between BIS values and FeS or calculated concentrations of $\mathrm{P}$ were modeled by nonlinear regression, using XLSTAT Version 2011.4.04, Addinsoft.

To calculate the $\mathrm{EC} \mathrm{BO}_{\mathrm{BIS}}$ corresponding to half-maximal effect (BIS 50), the data were fitted to a dose-response curve using GraphPad Prism version 4.03 for windows, (GraphPad Software, San Diego, CA). A semilogarithmic plot of propofol or sevoflurane concentrations versus BIS was generated, and data were fit to an inhibitory sigmoid Emax model. In accordance with the clinical use of BIS, EO was constrained to 98 and Emax was constrained to 0 . The Hill Slope was variable and optimized to get the best fit.

The $\mathrm{EC} \mathrm{BO}_{\mathrm{BIS}}$ of propofol was calculated using all steady-state peri-

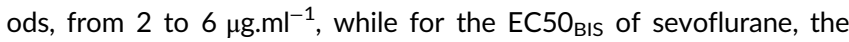
steady-state periods at Fe $5 \%$ were excluded to allow calculation. We excluded these periods because $\mathrm{EC} \mathrm{BO}_{\mathrm{BIS}}$ calculation was performed using the inhibitory sigmoid Emax model, which implies a specific relationship between the dose of the drug and the measured effect: increasing doses must be associated with an increasing effect, until a maximal effect is reached. Under $5 \%$ sevoflurane, we observed a paradoxical increase of the bispectral index: consequently, EC50 calculation was performed in the range of concentrations fitting the Emax model, from $0 \%$ to $4 \%$ (included) of sevoflurane. The values of $\mathrm{EC} 5 \mathrm{~B}_{\mathrm{BIS}}$ were estimated, with $95 \%$ confidence.

Therefore, we were able to compare both agents at equipotent levels of BIS depression. Equipotency was defined as similar multiple of $\mathrm{EC} 50_{\mathrm{BIS}} \pm 0.1 \mathrm{EC} \mathrm{BO}_{\mathrm{BIS}}$ :

Spectral data were compared between sevoflurane and propofol, at equipotent level of BIS depression, using a Mann-Whitney test. Incidence of EEG epileptoid signs and burst suppression were compared at equipotent level of BIS depression, using a Fisher exact test. A value of $P<0.05$ was considered as significant.

The sample size was calculated from the minimal number of recordings required at each steady-state period to allow comparison between sevoflurane and propofol at equipotent levels of BIS depression. In order to detect a difference of mean of $30 \%$, with an expected standard deviation of $25 \%$, and a statistical power of $0.9,11$ recordings at each steady-state period were required. Each patient providing two recordings, the minimal number of patients to include was 66; we chose to include 73 patients $(+10 \%)$ to control for the possible loss of data due to technical problem of data acquisition or analysis.

\section{3 | RESULTS}

Seventy-three patients (11 [5-18] years old, $41 \pm 15 \mathrm{~kg}$ ) were included in the study; 37 in group $\mathrm{P}$ and 36 in group S. Fifty-one steady-state periods were successfully recorded in the group $P$, and fifty-two in group S. Thus, a total of 103 steady-state periods were analyzed.

No patient showed clinical seizures whatever the anesthetic agent. No patient reported any element in favor of a possible awareness.

\section{1 | BIS-concentration relationships}

Under propofol, BIS decreased monotonically as propofol concentrations increased from 0 to $6 \mu \mathrm{g} \cdot \mathrm{ml}^{-1}$. Using nonlinear regression, the best fit to model the BIS-propofol concentration relationship was a second-order polynomial regression: $y=0.903 x^{2}-18.35 x+96.11$, $r^{2}=0.963(P<0.0001)$

Using the Emax dose-response curves, calculated from 0 to $6 \mu \mathrm{g} \cdot \mathrm{ml}^{-1}$ of propofol concentrations, the $\mathrm{EC}^{5} 0_{\mathrm{BIS}}$ was $3.3 \mu \mathrm{g} \cdot \mathrm{ml}^{-1}$.

Under sevoflurane, BIS values followed a different profile characterized by a dose-dependent decrease from $0 \%$ to $4 \%$ and then a paradoxical rise of the BIS values from $4 \%$ to $5 \%$. Using nonlinear regression, the best fit to model the BIS-sevoflurane concentration relationship was a third-order polynomial regression: $y=0.609 x^{3}+$ $1.073 x^{2}-30.23 x+94.93, r^{2}=0.955(P<0.0001)$.

Using the Emax dose-response curves, calculated from $0 \%$ to $4 \%$ of sevoflurane, the $\mathrm{EC} 50_{\mathrm{BIS}}$ was $2.1 \%(2.0 \%-2.3 \%, 95 \% \mathrm{Cl})$.

The nonlinear regression curves are represented on the Figure 2, where each concentration of propofol or sevoflurane is expressed as a multiple of the respective calculated $\mathrm{EC} 5 \mathrm{~B}_{\mathrm{BIS}}$. 


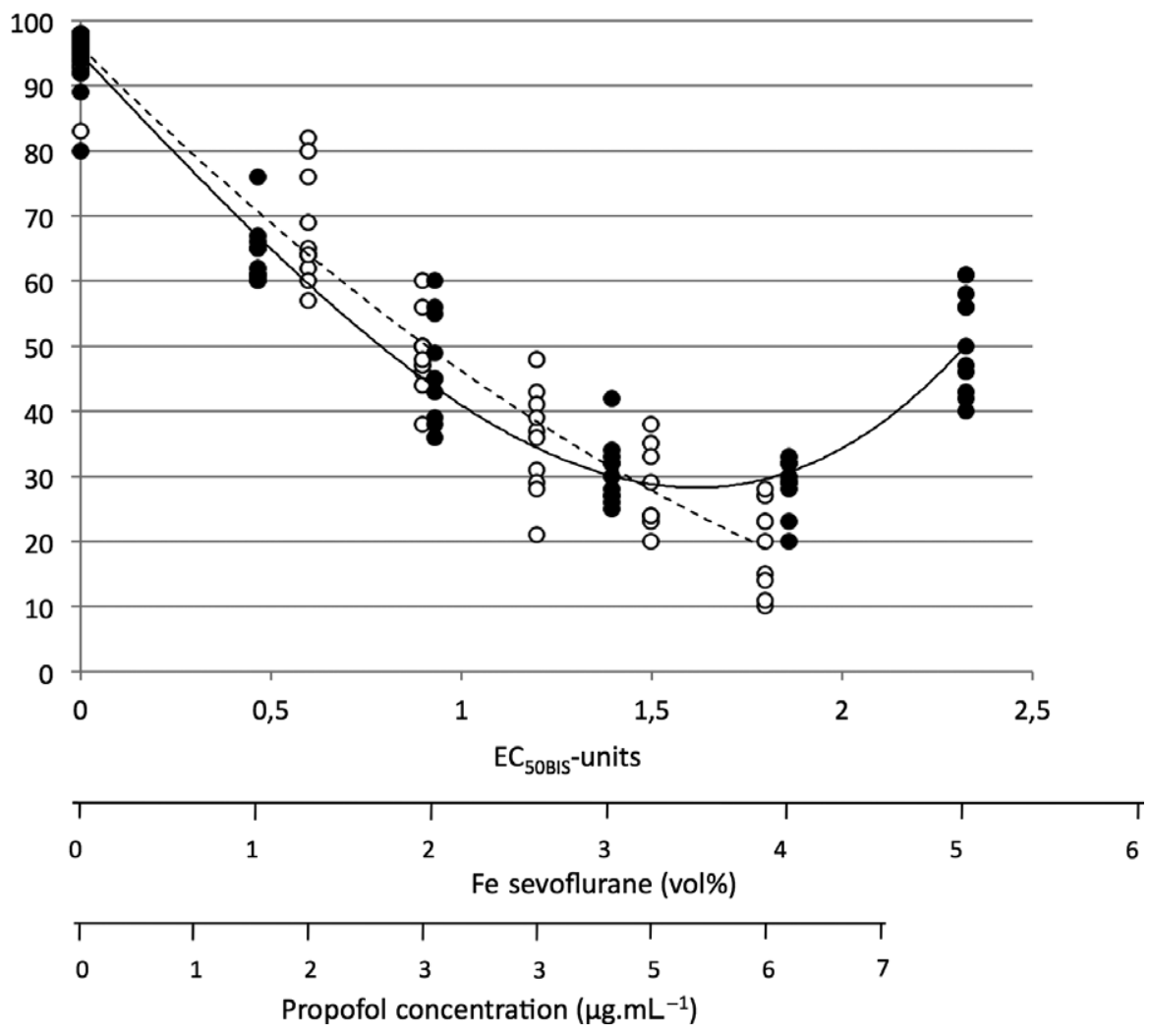

FIGURE 2 BIS-concentration relationships in children under sevoflurane and propofol:the nonlinear regression curves are represented with concentrations expressed as a multiple of the respective calculated $\mathrm{EC} \mathrm{O}_{\mathrm{BIS}}$ (corresponding to halfmaximal effect at BIS 50). Black line and filled dots: Sevoflurane group. Dotted line and white dots: Propofol group
Using the $E C 50_{\mathrm{BIS}}$ as unit, we expressed each concentration associated with a steady-state period, as a multiple of the respective $\mathrm{EC} 50_{\mathrm{BIS}}$ (Figure 2 and Tables 1, 2).

\section{2 $\quad$ Raw EEG and epileptiform signs}

Under propofol, the EEG changes associated with increasing concentrations were basically characterized by more and more delta waves associated with periods of burst suppression especially occurring at 5 and $6 \mu \mathrm{g} \cdot \mathrm{ml}^{-1}$. No patient showed major ES; only some minor isolated ES were observed during burst suppression periods.

Under sevoflurane, the EEG changes associated with increasing concentrations appeared as biphasic: at low concentrations, EEG showed as expected slow oscillations; however, from 4\%, the EEG traces showed faster oscillations including frequent rhythmic polyspikes and sometimes periodic epileptiform discharges occurring most often at $5 \%$ of $\mathrm{FeS}$.

The incidence of minor ES, major ES, and burst suppressions is described in Table 1.

When compared at equipotent BIS level inhibition, burst suppressions occurred more frequently under propofol at $1.8 \mathrm{EC}^{\mathrm{B} \mathrm{BIS}_{\mathrm{BS}}}$ $(P=0.0007)$.

\section{3 | Spectral analysis of raw EEG}

All spectral data of EEG are presented on the Table 2.

Under propofol, the lowest concentration $\left(2 \mu \mathrm{g} \cdot \mathrm{ml}^{-1}\right)$ was characterized by an increase of percentage of beta oscillations compared to baseline. As expected, with the deepening of anesthesia, the relative contribution of delta oscillations increased.

Under sevoflurane, the lowest concentration (1\%) was also characterized by an increase of the percentage of beta oscillations compared to baseline. At 2, 3, 4, and 5\% of sevoflurane, the slowing down of the EEG was associated with an increase of the theta waves rather than the expected increase of delta waves. An increase of the beta waves occurred at $5 \%$ of sevoflurane.

Spectral components of EEG were compared at similar or close levels of BIS depression expressed as multiple of $\mathrm{EC} 50_{\mathrm{BIS}}$. At 1.4

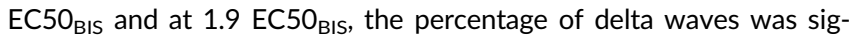
nificantly lower under sevoflurane compared to propofol (Table 2).

\section{DISCUSSION}

In a wide range of concentrations, we have shown that under propofol, the BIS decreased as the concentrations increased, while under sevoflurane the BIS decreased for increasing concentrations from $0 \%$ to $4 \%$, and then increased when sevoflurane concentration reached $5 \%$. Visual assessment of raw EEG traces and spectral analysis revealed that increasing concentrations of sevoflurane and propofol were characterized by different electroencephalographic profiles.

The BIS provides a single number from 100 (awake) to 0 (very deep anesthesia), resulting from an automated calculation based on frontal EEG parameters. The algorithm of calculation was validated against a sedation score measured in adults receiving intravenous or inhaled anesthetic agents. In children, the validity of the BIS has been questioned. However, the BIS value is only the result of an 
TABLE 1 Incidence of minor epileptoid signs (ES), major ES, and burst suppression periods at each concentration of propofol and sevoflurane. Incidences are expressed as number (percentage)

\begin{tabular}{|c|c|c|c|c|c|c|c|}
\hline PROPOFOL ( $\mu \mathrm{g} / \mathrm{ml})$ & 0 & 2 & 3 & 4 & 5 & 6 & \\
\hline BIS depression level (multiple of $\mathrm{EC} \mathrm{O}_{\mathrm{BIS}}$ ) & 0 & 0.6 & 0.9 & 1.2 & 1.5 & 1.8 & \\
\hline Number of analyzed steady-state periods & 51 & 11 & 11 & 11 & 8 & 10 & \\
\hline Major ES & $0(0)$ & $0(0)$ & $0(0)$ & $0(0)$ & $0(0)$ & $0(0)$ & \\
\hline Burst suppression & $0(0)$ & $0(0)$ & $0(0)$ & $1(9)$ & $1(13)$ & $8(80)$ & \\
\hline SEVOFLURANE (vol \%) & 0 & 1 & 2 & & 3 & 4 & 5 \\
\hline BIS depression level (multiple of $\mathrm{EC} 50_{\mathrm{BIS}}$ ) & 0 & 0.5 & 0.9 & & 1.4 & 1.9 & 2.3 \\
\hline Number of analyzed steady-state periods & 52 & 11 & 10 & & 11 & 9 & 11 \\
\hline Minor ES & $0(0)$ & $0(0)$ & $1(10)$ & & $1(9)$ & $4(44)$ & $11(100)$ \\
\hline Major ES & $0(0)$ & $0(0)$ & $0(0)$ & & $1(9)$ & $1(11)$ & $10(91)$ \\
\hline
\end{tabular}

Gray columns correspond to equipotent BIS suppression levels (multiples of $\mathrm{EC} \mathrm{SO}_{\mathrm{BIS}}$ ) between one concentration of propofol and one concentration of sevoflurane.

TABLE 2 Spectral analysis of EEG traces at each concentration of propofol and sevoflurane spectral power of delta, theta, alpha, and beta bands, expressed as percentage of Total Spectral Power (mean \pm SD)

\begin{tabular}{|c|c|c|c|c|c|c|c|}
\hline PROPOFOL ( $\mu \mathrm{g} / \mathrm{ml})$ & 0 & 2 & 3 & 4 & 5 & 6 & \\
\hline BIS depression level (multiple of $\mathrm{EC} 5 \mathrm{~B}_{\mathrm{BIS}}$ ) & 0 & 0.6 & 0.9 & 1.2 & 1.5 & 1.8 & \\
\hline Delta (\%) & $64 \pm 16$ & $49 \pm 27^{\S}$ & $55 \pm 15$ & $79 \pm 14$ & $88 \pm 10^{\#}$ & $94 \pm 4 * * *$ & \\
\hline Alpha (\%) & $7 \pm 4$ & $8 \pm 6$ & $15 \pm 7$ & $9 \pm 6$ & $6 \pm 7$ & $2 \pm 1^{*}$ & \\
\hline Beta (\%) & $16 \pm 11$ & $37 \pm 29^{\S}$ & $21 \pm 11^{\dagger}$ & $6 \pm 4$ & $3 \pm 2$ & $1 \pm 1$ & \\
\hline $\mathrm{BIS}$ depression level (multiple of $\mathrm{EC} 5 \mathrm{O}_{\mathrm{BIS}}$ ) & 0 & 0.5 & 0.9 & & 1.4 & 1.9 & 2.3 \\
\hline Delta (\%) & $63 \pm 15$ & $15 \pm 7$ & $62 \pm 15$ & & $68 \pm 14$ & $60 \pm 17$ & $58 \pm 8$ \\
\hline Theta (\%) & $11 \pm 5$ & $8 \pm 10$ & $18 \pm 5$ & & $17 \pm 8$ & $25 \pm 13$ & $18 \pm 10$ \\
\hline Alpha (\%) & $6 \pm 4$ & $11 \pm 6$ & $11 \pm 7$ & & $9 \pm 5$ & $7 \pm 6$ & $9 \pm 4$ \\
\hline
\end{tabular}

Gray columns correspond to equipotent BIS suppression levels between one concentration of propofol and one concentration of sevoflurane. Comparisons between the propofol group and the sevoflurane group were only performed at equipotent BIS suppression levels.

Differences between propofol $6 \mu \mathrm{g} \cdot \mathrm{ml}^{-1}(1.8 \mathrm{EC} 50)$ and sevoflurane $4 \%$ (1.9 EC50): *P<0.05; ***P<0001.

Differences between propofol $5 \mu \mathrm{g} \cdot \mathrm{ml}^{-1}$ (1.5 EC50) and sevoflurane 3\% (1.4 EC50): \#P< 0.05 ; \#\#\# < 0001.

Differences between propofol $3 \mu \mathrm{g} \cdot \mathrm{ml}^{-1}(0.9 \mathrm{EC} 50)$ and sevoflurane $2 \%(0.9 \mathrm{EC} 50): \dagger P<0.05 ; \dagger \dagger P<0.01$.

Differences between propofol $2 \mu \mathrm{g} \cdot \mathrm{ml}^{-1}$ (0.6 EC50) and sevoflurane 1\% (0.5 EC50): $\S P<0.05$.

automated calculation, so any difference in BIS profiles between adults and children might reflect differences in the effects of anesthetics, rather than in the accuracy of the device.

Most GABAergic anesthetics induce a dose-dependent slowing down of the EEG oscillations, reaching periods of isoelectric trace reflecting total cortical inhibition (burst suppression). The relationship between BIS and propofol concentration seems to follow this principle. Indeed, in our study, we found that under propofol, BIS values decreased monotonically as the propofol concentrations increased from 2 to $6 \mu \mathrm{g} \cdot \mathrm{ml}^{-1}$. Our results are in agreement with several adult $^{16-18}$ and pediatric ${ }^{5,19}$ studies.
Using spectral analysis, we demonstrated that in children over 5 years old, the EEG effects of propofol were close to those observed in adults. The EEG spectral profile during "surgical anesthesia" (propofol $117.2 \pm 26 \mu \mathrm{g} \cdot \mathrm{kg}^{-1} \cdot \mathrm{min}^{-1}$ ) was previously described, ${ }^{20}$ with dominant slow activity (delta). Our results confirm this pattern. In addition, we have demonstrated that this increase in delta activity was dose-dependent. We have also described a dynamic profile of rapid beta oscillations: beta activity increased at low propofol concentrations (2$3 \mu \mathrm{g} \cdot \mathrm{ml}^{-1}$ ), and was markedly decreased for higher concentrations.

Some case reports have suggested a possible association between propofol administration and clinical seizures, but they did 
not include EEG data. ${ }^{21,22}$ Using visual assessment of raw EEG, our results demonstrated that in our population, the epileptogenic potential of propofol was negligible, even at the highest concentrations. We only observed rare isolated spikes occurring during periods of burst suppression. In addition, we have evidenced that the high incidence of burst suppression at high concentrations was the main characteristic of the propofol EEG effects. Our results are in agreement with the study of Jaaskelainen, performed in healthy adults. $^{6}$

In our population, BIS profile under sevoflurane was biphasic. The first phase was a dose-dependent decrease of BIS when sevoflurane concentrations increased from $0 \%$ to $4 \%$. Similar findings have been reported in previous studies. ${ }^{7,23-25}$ The second phase was a reascension of the BIS when sevoflurane concentrations increased from $4 \%$ to $5 \%$. These paradoxical changes of the BIS in children receiving high concentrations of sevoflurane were already suggested in two pediatric studies, but the authors failed to explain this phenomenon. ${ }^{7,8}$ An increase in the BIS when isoflurane concentration increased from $0.8 \%$ to $1.6 \%$ was also reported in adults. The authors suggested that the increase in BIS might be related to continuous pre-burst EEG patterns consisting of high-frequency activity, without being able to confirm this by raw EEG analysis. ${ }^{26}$

The spectral analysis of the EEG traces allowed us to understand the changes associated with sevoflurane increasing concentrations. Akeju described the EEG spectral profile of children under sevoflurane "surgical anesthesia" (concentration $2.21 \pm 0.44 \%$ ), with a dominant slow activity in the delta band, and also in the theta band. ${ }^{3}$ Our results confirm this spectral profile. In addition, we have shown that the power increase in the delta and theta bands occurs when sevoflurane concentration reaches $2 \%$, and is almost immediately maximal. The dynamic profile of the beta waves under sevoflurane is particularly interesting. As for propofol, beta power increases at very low concentrations of sevoflurane (1\%), and then it significantly decreases for concentrations between $2 \%$ and $4 \%$. Under $5 \%$ of sevoflurane, however, we observed a substantial increase in beta power. The visual analysis of raw EEG provided additional information: under $5 \%$ of sevoflurane, rhythmic polyspikes occurred very frequently, overdriving the slow oscillations. These major ES led to a shift of the EEG waves toward high frequencies, as described in adults by Jaaskelainen. ${ }^{6}$ Finally, when processed by the BIS calculation algorithm, these fast waves result in a higher BIS value. Our findings suggest that the BIS increase under high concentrations of sevoflurane might be caused by the processing of major epileptoid signs through the algorithm of the device. Epileptoid signs may falsely elevate EEG-derived depth of anesthesia indices. From a clinical point of view, clinicians confronted to high BIS values under deep sevoflurane anesthesia $(\geq 3 \%)$ should consider decreasing $\mathrm{FeS}$ in order to avoid epileptoid activity, rather than think anesthesia is too light and increase FeS to obtain lower BIS values.

The occurrence of EEG epileptoid signs has been described during induction and maintenance of sevoflurane anesthesia. In adults, the incidence of major ES is considered as dose-dependent. We previously demonstrated that the minimal alveolar concentration of sevoflurane associated with major ES in children was between $4 \%$ and $5 \%{ }^{2}$ Our current results confirm that from $4 \%$ of sevoflurane, epileptoid signs may be expected. The physiopathology of these epileptiform discharges remains unclear and up to now the potential morbidity of major ES is unknown. However, given the very high number of sevoflurane anesthetics performed since the release of this halogenated agent, we can assume that the long-term consequences of epileptoid signs, if they exist, are not of major importance. It might be interesting to make a connection between our results and a study based on proton magnetic resonance spectroscopy performed under sevoflurane versus propofol anesthesia. The authors report higher glucose and lactate in the human brain, and an enhanced neuronal activity under sevoflurane compared to propofol. In addition, they describe a positive relationship between brain lactate elevation and an agitation score upon emergence. ${ }^{27}$

As we studied a wide range of sevoflurane and propofol concentrations, which were not strictly equipotent, the design of our study made hazardous comparison between propofol and sevoflurane. The only comparison that we have allowed was calculated at equipotent level of BIS depression. Unfortunately with this approach, there was no equipotent propofol concentration corresponding to the highest concentration of sevoflurane. So, we could not strictly conclude that sevoflurane was associated with a higher incidence of epileptoid signs, as it has been previously demonstrated in adults.

In conclusion, our study described EEG patterns in children anesthetized with propofol or sevoflurane. In the studied range of concentrations, we demonstrated that propofol induced a dosedependent cortical inhibition reflected by a steady decrease in BIS, while high concentrations of sevoflurane were rather associated with EEG activation and epileptoid signs, which may be responsible for an apparently paradoxical increase in BIS. These findings may help the clinicians to more accurately adapt intraoperative administration of anesthetics in children.

\section{ETHICAL APPROVAL}

This study was approved by our local Ethics Committee: CPP Saint Antoine, Paris, France (Approval number 04605). The study was conducted in 2005-2006 and at this time registration was not mandatory. We registered this trial on January 21, 2016 and completed on September 4, 2016 (ClinicalTrials.gov ID: NCTO2893904).

\section{CONFLICT OF INTEREST}

The authors declare no competing interests in the subject or materials discussed in this manuscript.

\section{ORCID}

Agnes Rigouzzo iD https://orcid.org/0000-0001-5865-3168

Dominique Laude iD https://orcid.org/0000-0003-3097-9849 


\section{REFERENCES}

1. Vakkuri A, Yli-Hankala A, Särkelä $M$, et al. Sevoflurane mask induction of anaesthesia is associated with epileptiform EEG in children. Acta Anaesthesiol Scand. 2001;45(7):805-811.

2. Gibert S, Sabourdin N, Louvet N, et al. Epileptogenic effect of sevoflurane: determination of the minimal alveolar concentration of sevoflurane associated with major epileptoid signs in children. Anesthesiology. 2012;117(6):1253-1261.

3. Akeju O, Pavone KJ, Thum JA, et al. Age-dependency of sevoflurane-induced electroencephalogram dynamics in children. $\mathrm{Br} J$ Anaesth. 2015;115(Suppl 1):i66-i76.

4. Lee JM, Akeju O, Terzakis K, et al. A prospective study of age-dependent changes in propofol-induced electroencephalogram oscillations in children. Anesthesiology. 2017;127(2):293-306.

5. Rigouzzo A, Girault L, Louvet N, et al. The relationship between bispectral index and propofol during target-controlled infusion anesthesia: a comparative study between children and young adults. Anesth Analg. 2008;106(4):1109-1116, table of contents.

6. Jääskeläinen SK, Kaisti K, Suni L, Hinkka S, Scheinin H. Sevoflurane is epileptogenic in healthy subjects at surgical levels of anesthesia. Neurology. 2003;61(8):1073-1078.

7. Denman WT, Swanson EL, Rosow D, Ezbicki K, Connors PD, Rosow CE. Pediatric evaluation of the bispectral index (BIS) monitor and correlation of BIS with end-tidal sevoflurane concentration in infants and children. Anesth Analg. 2000;90(4):872-877.

8. Kim HS, Oh AY, Kim CS, Kim SD, Seo KS, Kim JH. Correlation of bispectral index with end-tidal sevoflurane concentration and age in infants and children. Br J Anaesth. 2005;95(3):362-366.

9. Davidson AJ, Wong A, Knottenbelt G, Sheppard S, Donath S, Frawley G. MAC-awake of sevoflurane in children. Paediatr Anaesth. 2008;18(8):702-707.

10. McKeever S, Johnston L, Davidson AJ. Sevoflurane-induced changes in infants' quantifiable electroencephalogram parameters. Paediatr Anaesth. 2014;24(7):766-773.

11. Särkelä M, Ermes MJ, van Gils MJ, Yli-Hankala AM, Jäntti VH, Vakkuri AP. Quantification of epileptiform electroencephalographic activity during sevoflurane mask induction. Anesthesiology. 2007;107 (6):928-938.

12. Kataria BK, Ved SA, Nicodemus HF, et al. The pharmacokinetics of propofol in children using three different data analysis approaches. Anesthesiology. 1994;80(1):104-122.

13. Schnider TW, Minto CF, Shafer SL, et al. The influence of age on propofol pharmacodynamics. Anesthesiology. 1999;90(6):15021516.

14. Yli-Hankala A, Vakkuri A, Särkelä $M$, Lindgren L, Korttila K, Jäntti V. Epileptiform electroencephalogram during mask induction of anesthesia with sevoflurane. Anesthesiology. 1999;91(6):1596-1603.

15. Rampil IJ. A primer for EEG signal processing in anesthesia. Anesthesiology. 1998;89(4):980-1002.

16. Glass PS, Bloom M, Kearse L, Rosow C, Sebel P, Manberg P. Bispectral analysis measures sedation and memory effects of propofol, midazolam, isoflurane, and alfentanil in healthy volunteers. Anesthesiology. 1997;86(4):836-847.

17. Leslie K, Sessler DI, Schroeder M, Walters K. Propofol blood concentration and the Bispectral Index predict suppression of learning during propofol/epidural anesthesia in volunteers. Anesth Analg. 1995;81 (6):1269-1274.

18. Doi M, Gajraj RJ, Mantzaridis H, Kenny GN. Relationship between calculated blood concentration of propofol and electrophysiological variables during emergence from anaesthesia: comparison of bispectral index, spectral edge frequency, median frequency and auditory evoked potential index. Br J Anaesth. 1997;78(2):180-184.

19. Jeleazcov $\mathrm{C}$, Ihmsen $\mathrm{H}$, Schmidt J, et al. Pharmacodynamic modelling of the bispectral index response to propofol-based anaesthesia during general surgery in children. Br J Anaesth. 2008;100(4):509-516.

20. Akeju O, Westover MB, Pavone KJ, et al. Effects of sevoflurane and propofol on frontal electroencephalogram power and coherence. Anesthesiology. 2014;121(5):990-998.

21. Borgeat A, Dessibourg C, Popovic V, Meier D, Blanchard M, Schwander D. Propofol and spontaneous movements: an EEG study. Anesthesiology. 1991;74(1):24-27.

22. Reddy RV, Moorthy SS, Dierdorf SF, Deitch RD, Link L. Excitatory effects and electroencephalographic correlation of etomidate, thiopental, methohexital, and propofol. Anesth Analg. 1993;77 (5):1008-1011.

23. Davidson AJ, Huang GH, Rebmann CS, Ellery C. Performance of entropy and Bispectral Index as measures of anaesthesia effect in children of different ages. Br J Anaesth. 2005;95(5):674-679.

24. Degoute CS, Macabeo C, Dubreuil C, Duclaux R, Banssillon V. EEG bispectral index and hypnotic component of anaesthesia induced by sevoflurane: comparison between children and adults. $\mathrm{Br} J$ Anaesth. 2001;86(2):209-212.

25. McCann ME, Bacsik J, Davidson A, Auble S, Sullivan L, Laussen P. The correlation of bispectral index with endtidal sevoflurane concentration and haemodynamic parameters in preschoolers. Paediatr Anaesth. 2002;12(6):519-525.

26. Detsch O, Schneider G, Kochs E, Hapfelmeier G, Werner C. Increasing isoflurane concentration may cause paradoxical increases in the EEG bispectral index in surgical patients. Br J Anaesth. 2000;84 (1):33-37.

27. Jacob Z, Li H, Makaryus R, et al. Metabolomic profiling of children's brains undergoing general anesthesia with sevoflurane and propofol. Anesthesiology. 2012;117(5):1062-1071.

How to cite this article: Rigouzzo A, Khoy-Ear L, Laude D, et al. EEG profiles during general anesthesia in children: $A$ comparative study between sevoflurane and propofol. Pediatr Anaesth. 2019;29:250-257. https://doi.org/ 10.1111/pan.13579 